\title{
The look and feel of resilience: A qualitative study of physicians' perspectives
}

\author{
Alicia J Polachek ${ }^{1}$, Jean E Wallace ${ }^{2}$, Mamta Gautam ${ }^{3}$, Jill A de Grood ${ }^{1}$, Jane B Lemaire*1,4 \\ ${ }^{1}$ W21C Research and Innovation Centre, University of Calgary, Calgary, Alberta, Canada \\ ${ }^{2}$ Department of Sociology, University of Calgary, Calgary, Alberta, Canada \\ ${ }^{3}$ Department of Psychiatry, University of Ottawa, Ottawa, Ontario, Canada \\ ${ }^{4}$ Department of Medicine, Division of General Internal Medicine, University of Calgary, Calgary, Alberta, Canada
}

Received: November 5, 2015

DOI: $10.5430 /$ jha.v5n2p47
Accepted: December 20, 2015 Online Published: January 4, 2016

URL: http://dx.doi.org/10.5430/jha.v5n2p47

\begin{abstract}
Some physicians can effectively cope and thrive in the face of potentially stressful job conditions, while others experience serious, negative consequences. This ability to be resilient may improve physician wellness and benefit health care organizations, yet little is known about what resilience means to physicians. This paper explores how physicians understand resilience both as they observe it in their colleagues and as they experience it themselves. Semi-structured interviews were conducted with 32 physicians practicing in Alberta, Canada. Two questions explored physicians' experiences of resilience or non-resilience, while two other questions considered what they observed in physician colleagues. Interview transcripts were independently coded by two authors and discussed to ensure agreement on the key themes. There were several similarities in how physicians described resilience or non-resilience in themselves and their colleagues related to control, positivity or negativity, boundaries and balance, coping, and support. There were also important differences in how physicians described their own experiences and their observations of colleagues. Participants' portrayals of themselves suggested being immune to stress and responsible for their success or failure in being resilient. Their depictions of colleagues, however, focused on professionalism, work performance, commitment to values, and experience or wisdom. There appears to be a difference in how physicians understand resilience in themselves compared to what they observe in their colleagues. Specifically, physicians may hold unrealistic and unachievable expectations for their own resilience. Initiatives aimed at improving physician resilience and wellness may be best served by raising awareness about more realistic expectations and self-appraisals.
\end{abstract}

Key Words: Physician resilience, Workplace stress, Culture of medicine, Physician wellness

\section{INTRODUCTION}

Physicians experience extensive work stress ${ }^{[1-3]}$ and a higher risk for burnout, ${ }^{[4-7]}$ depression, ${ }^{[8]}$ and suicide ${ }^{[9,10]}$ compared to the general population. Conditions such as burnout can have serious consequences not only for individual physicians, but also for health care organizations. ${ }^{[6,7,11]}$ For example, burnout may contribute to medical mistakes such as diagnos- tic errors or prescription of incorrect medication, poor quality patient care, poor interactions with patients, reduced productivity, difficulty in making decisions, poor communication, irritability, decreased focus or organizational commitment, and staff turnover, all of which can impact the health care organization as well. ${ }^{[6,7,11,12]}$

Physicians' work is often characterized by a high work-

\footnotetext{
* Correspondence: Jane B Lemaire; Email: lemaire@ucalgary.ca; Address: Department of Medicine, Division of General Internal Medicine, Health Sciences Centre, 3330 Hospital Drive NW, Calgary, Alberta T2N 4N1, Canada.
} 
load, ${ }^{[6,11,13]}$ long workhours, ${ }^{[6]}$ and high cognitive and emotional demands ${ }^{[11,14]}$ where physicians are likely to encounter stressful or traumatic events involving death or serious injury. ${ }^{[14,15]}$ Despite this demanding and stressful work environment, many physicians are able to successfully cope and even thrive, experiencing joy, meaning, and satisfaction from their work. ${ }^{[16]}$ Some physicians appear to be more resilient in the face of potentially stressful work conditions as a result of intrinsic or personality factors. ${ }^{[17]}$ This resiliency may have the potential to improve physician wellness by mitigating distress, especially when used as a prevention strategy rather than a response to existing problems. ${ }^{[18,19]}$ Furthermore, since organizational characteristics such as work hours and demands may be difficult to change, improving physicians' resilience may hold benefits for both individual physicians and the health care system more broadly. ${ }^{[17]}$ These stakeholders may be well served by mounting a joint effort at fostering physician resilience. ${ }^{[11,17,20]}$ Before implementing programs to improve physician resilience, it is important to understand what resilience means to physicians in order to develop programs that resonate with physicians and encourage their participation in such programs.

There is little agreement about how to define resilience, ${ }^{[21,22]}$ and current understandings focus on distressed or vulnerable groups including youth or adolescents, ${ }^{[23-25]}$ single mothers, ${ }^{[26]}$ immigrants, ${ }^{[27]}$ the elderly, ${ }^{[28]}$ and the seriously ill. ${ }^{[29]}$ While some characteristics of resilience identified in these groups may translate to physicians, there are likely also important differences between these populations. Physician resilience may differ from resilience in other groups, and different strategies may be required for improving physicians' ability to cope and thrive in their work environment.

Research has begun to consider resilience in medical trainees and physicians. In one study of medical trainees, resilience involved acclimating to traumas experienced during clinical training. ${ }^{[15]}$ Studies describing attributes of resilient physicians highlighted attitudes and perspectives, balance and prioritization, practice management style, supportive relationships, job-related gratification, and practices and routines. ${ }^{[16,30]}$ Finally, studies of resiliency training ${ }^{[21]}$ discussed the importance of understanding the effects of stress and developing resiliency. ${ }^{[31]}$ Suggested strategies included adaptability, teamwork, rebounding from stress, refocusing and reframing, mindful self-compassion, and self-awareness, among others. ${ }^{[19,32-34]}$

Despite these studies, there remains knowledge gaps in understanding physician resilience, particularly in terms of what it means to physicians themselves. Better understanding this conceptualization may assist with efforts aimed at improving physician resilience and encouraging physician engagement in such efforts. If programming does not resonate with physicians, it is unlikely that they will actively participate in these endeavours. This paper therefore explores how physicians understand resilience both as they observe it in their colleagues and as they experience it themselves.

\section{Methods}

\subsection{Study design and data collection}

Data were obtained from a qualitative study of physicians practicing in Alberta, Canada. A voluntary non-probability sampling strategy was used. In line with recommendations for non-probabilistic qualitative research, a sample size of 30 was estimated as sufficient to achieve theoretical saturation where no new themes emerged, without being too large for detailed analysis of individual physicians' perceptions and experiences. ${ }^{[35,36]}$ Using a random number generator, potential participants were identified from a list of all physicians registered with the Alberta College of Physicians and Surgeons in March 2011. These physicians were sent mail invitations asking them if they would be interested in volunteering for an interview. Electronic invitations were also sent when email addresses were available. This process of recruitment continued until our desired sample size was reached. In total, 460 physicians were contacted and 32 volunteered to participate. No participants subsequently withdrew from the study. Prior to the interview, participants were informed that the aim of the study was to explore how physicians define and conceptualize resilience, with the hope that the information obtained could not only help in understanding physician resilience and how it could be measured, but also that it may help in identifying strategies to enhance resilience, promote physician wellness, and prevent burnout.

A female sociologist (JD) with experience and training in qualitative research conducted one-on-one, semi-structured telephone interviews between July and December 2011. She did not have existing relationships with the participants before the interviews. The average interview length was 19 minutes (range $=9-37$ ). Each participant completed a single interview and took the call at a location of their choosing. Interviews were digitally recorded, transcribed verbatim, and assigned an identification number. Field notes were not utilized.

Interview questions centred on having physician participants define, describe, and provide examples of what resilience means to them in the context of their work. This paper focuses specifically on four interview questions that explore the look and the feel of physician resilience, as outlined in Table 1. The look refers to how participants conceptualized resilience when asked to describe their colleagues, and the 
feel refers to how participants conceived of resilience when asked to describe their own experiences. While the initial aim was not to compare how physicians understand resilience in themselves compared to how they perceive it in others, it is possible that participants may provide different information when asked about their colleagues rather than themselves. Therefore, questions were asked about physicians' own experiences as well as what they saw in their colleagues in order to develop a comprehensive understanding of what resilience means to physicians. During analysis, it became clear that physicians may hold different understandings or expectations of resilience in themselves compared to their colleagues, and the look and the feel therefore became useful analytic categories for describing physicians' varying conceptualizations of resilience.

Table 1. Interview questions used to explore the look and the feel of physician resilience or non-resilience

\begin{tabular}{ll}
\hline The Look & The Feel \\
\hline “Think of a physician colleague whom you consider resilient. & $\begin{array}{l}\text { "Think back to an example of a time or situation when you felt } \\
\text { you were resilient. How would you describe the feeling of } \\
\text { What is it that you see in them that signals their resiliency to } \\
\text { you?" }\end{array}$ \\
$\begin{array}{l}\text { "Think of a physician colleague whom you do not consider very } \\
\text { resilient. What is it that you see in them that signals to you that } \\
\text { they are not very resilient?” }\end{array}$ & $\begin{array}{l}\text { you were not very resilient. What did it feel like being } \\
\text { unresilient?” }\end{array}$ \\
\hline
\end{tabular}

\subsection{Participants}

The sample included 16 women and 16 men. The majority of participants were married (69\%) and had children (75\%). Their average age was 49 years (range $=31-83$ ) and the average length of practice was 20 years (range $=3-58$ ). Various medical contexts were represented, with $50 \%$ of participants from a medical specialty, $37 \%$ from primary care, and $13 \%$ from a surgical specialty. Participants worked in various practice settings: academic (28\%), group with shared office space and expenses (28\%), solo with shared call (10\%), solo $(6 \%)$, or other $(28 \%)$ such as hospital or community group practice. Most participants (81\%) took call, spending an average of 9 days per month on call (range $=0.5-24$ ). The type of remuneration included fee for service (41\%), alternative payment plans (28\%), combined methods (25\%, e.g., fee for service and flat private fee, fee for service and on call stipends), salary (3\%, e.g., university, professional corporation), and unknown (3\%). Detailed information on non-responders was not available.

\subsection{Data analysis}

Using an inductive strategy, two authors (AP and JD) independently reviewed the interview transcripts to identify themes that emerged from the data, rather than relying on pre-established categories. During open coding these authors read the transcripts to identify initial codes, before conducting selective coding where they re-read the transcripts in greater depth to refine and integrate themes. ${ }^{[37]}$ The authors then compared their analyses and discussed discrepancies to ensure agreement on the key themes. No analytic software was used, and participants were not provided with transcripts or asked for feedback on the findings.

\section{Results}

In comparing the look of resilience or non-resilience (i.e. how participants viewed this in their colleagues) to the feel of these experiences (i.e. how participants perceived this in themselves), the similarities are discussed before examining the differences. Key themes are outlined in Table 2. Several participant quotations that exemplify these themes are included in the following section, with identification numbers in brackets.

\subsection{The similarities}

Participants discussed having positive emotions, the importance of boundaries and balance, the use of coping strategies, and being in control when describing both the look and the feel of resilience. Resilient colleagues were described as "know[ing] how to separate work from the rest of their lives, and they have developed over the years mechanisms to do that properly... they can actually cope better because this is just not a job, it is a vocation" (\#32). They were also described as "proactive and optimistic and not negative in response to stress" (\#3) as well as "in control of things" (\#6). Similarly, the feel of resilience was described as "incredible... [you] feel like you're in control... you know you were able to cope...then go home and be able to interact with your family in a very positive way" (\#22). These characteristics were highlighted in both the participants themselves and in their colleagues, suggesting that there may be some universal aspects of physician resilience that reflect personal and professional expectations or competencies. 
Table 2. Key themes describing the look and the feel of resilience or non-resilience

\begin{tabular}{|c|c|c|}
\hline & The Look & The Feel \\
\hline Resilience & $\begin{array}{l}\text { - Displaying positivity and optimism } \\
\text { - Maintaining boundaries or balance } \\
\text { - Having effective coping strategies } \\
\text { - Remaining in control } \\
\text { - Being committed to values and goals } \\
\text { - Remaining calm and level-headed } \\
\text { - Being expressive and friendly } \\
\text { - Having work-related success } \\
\text { - Having experience and wisdom }\end{array}$ & $\begin{array}{l}\text { - Experiencing positive emotions }{ }^{*} \\
\text { - Having balance in all aspects of their life } \\
\text { - Being able to cope } \\
\text { - Feeling in control } \\
\text { - Being immune to stress } \\
\text { - Bouncing back from stressful situations }\end{array}$ \\
\hline Non-Resilience & $\begin{array}{l}\text { - Having negative emotional/personality qualities } \\
\text { - Lacking support systems } \\
\text { - Displaying poor job performance } \\
\text { - Coping ineffectively } \\
\text { - Having poor health or unhealthy behaviours } \\
\text { - Lacking balance in aspects of their life }\end{array}$ & $\begin{array}{l}\text { - Experiencing negative emotions } \\
\text { - Lacking support } \\
\text { - Feeling out of control } \\
\text { - Being overwhelmed or stressed } \\
\text { - Being burnt out or lacking energy }\end{array}$ \\
\hline
\end{tabular}

${ }^{*}$ This indicates the themes that were identified for both the look and the feel of resilience or non-resilience

Participants described having negative emotions and lacking support when describing both the look and the feel of non-resilience. Non-resilient colleagues were described as "allow[ing] themselves to get depressed and morose" (\#27) in addition to having "social anxiety... [and] learning not to trust anybody. So lack of support. . . being a bit narcissistic" (\#14).

Similarly, one physician described the feel of non-resilience as being "vulnerable, there's a sense of fear... I think there can be some catastrophic thinking... [a] sense of despondency can creep in [and] a sense of confusion" (\#8). As another commented, "not having the support of my colleagues or the profession... made [getting through challenges] incredibly difficult" (\#16). Participants' descriptions again suggest several common attributes signalling non-resilience in themselves and their colleagues.

\subsection{The differences}

There were also important differences in how participants described the look of resilience or non-resilience compared to the feel. When asked about the look of resilience, participants described colleagues who were committed to values and goals, remained calm or level-headed, were expressive and friendly, had work-related success, and had experience or wisdom. One participant stated that resilient colleagues were "professional...good, competent physician[s]... They're... well read...fairly even keeled yet expressive and able to speak [their] mind" (\#3). Another described resilient colleagues as "being able to... focus on the big picture... so if something has happened...that's negative... they [see] an opportunity to... figure out where the problems are and to get better" (\#24). Participants focused on professionalism, good work performance, and job success when asked to describe resilient colleagues.

When asked to describe the feel of resilience in themselves, participants discussed being immune to or overcoming stresses. Physicians described "being confident that I would be able to overcome any difficulties in my path" (\#19) and involving "a sense of pride that I wouldn't let a situation like that put me under. . . if I really bounce back... not just pretending to be okay, but truly okay" (\#26). Participants also described being "able to surmount things that present, you can work through them" (\#17), you "plug on... [and] push through" (\#12) and you "overcome adversity" (\#24). Physicians' descriptions of their own resilience emphasized their ability to push through and rebound from stressful situations, suggesting they possessed an immunity or imperviousness to stress in a way that was not present when describing resilient colleagues.

When asked to describe the look of non-resilience, participants described colleagues who displayed poor job performance, coped ineffectively, had poor health or unhealthy behaviours, and lacked balance. One participant spoke of a colleague with "problems in their marriage [and] familyrelated issues" (\#32). The colleague was "either not coping with his work and is dumping everything on his family, or he... wants to compensate for not being able to cope by working really hard and getting away from his family" (\#32). Nonresilient colleagues were also viewed as "not as methodical in terms of handling many things... kind of sporadic... running around, not achieving very much versus the kind of more methodological resilient person who's able to function better in a high stress situation" (\#6). Participants again focused 
on job performance, balance, and coping behaviours, as they had when asked about colleagues' resilience, but here their descriptions of non-resilient colleagues focused on unsuccessful job performance, a lack of balance, and ineffective coping mechanisms, rather than the reverse.

When asked to describe the feel of non-resilience in themselves, participants focused on lacking control and being overwhelmed or burnt out by stress. One physician explained non-resilience as "a feeling of uncertainty and lack of confidence and loss of control, like you couldn't manage all the different aspects" (\#11) while another described it as "feeling insufficient energy to complete even common tasks and... that any new challenge would be a last straw" (\#19). Finally, they described non-resilience as feeling like "everything was stacked against me, and... getting through even a minute every day was more than I could cope with" (\#16). These descriptions of physicians' own non-resilience highlighted their inability to function and being overwhelmed by stress, suggesting that they felt responsible for not being immune to stresses.

\section{Discussion}

There were several similarities in how participants described resilience or non-resilience in their colleagues and how they described these experiences in themselves. Participants discussed being in control, displaying positivity and optimism, maintaining boundaries and balance, and having effective coping strategies when asked about both the look and then the feel of resilience. Furthermore, they discussed having negative emotions and a lack of support when asked about both the look and then the feel of non-resilience.

Perhaps more importantly, there were interesting differences in physicians' descriptions of the look of resilience or nonresilience compared to the feel. When discussing their own experiences, participants often portrayed themselves as impervious to stress and responsible for their success or failure in being resilient. Physicians reported being immune to stress, being able to push through stressful situations, and being able to bounce back from difficulties, as exemplified by one physician who described having to "just put your head down and do what needs to be done" (\#1).

This portrayal of being impervious to stress and responsible for their success or failure in being resilient was not emphasized when physicians described their colleagues. Rather, when asked about their colleagues, physicians focused on resiliency as maintaining balance and boundaries, developing coping strategies, and being committed to values and goals. Participants appeared to understand resilience in their colleagues as professionalism and success in their work per-

Published by Sciedu Press formance, rather than expecting them to be unaffected by stress. Participants also seemed to view their colleagues' incorporation of wellness into professionalism as an aspect of resilience.

These divergent perceptions suggest that physicians may understand and conceptualize resilience differently for themselves than for their colleagues. Resilience may mean different things in the context of physicians describing their own experiences compared to how they perceive it in their colleagues. That is, physicians may hold fundamentally different expectations or standards for themselves compared to their colleagues in terms of being resilient to workplace stresses and challenges. This is in line with psychological research suggesting that "cognition about the self differs in important ways from cognition about others" ${ }^{[38]}$ with regard to personality characteristics or traits, causal attributions of behaviour, and evaluations or appraisals. ${ }^{[38-40]}$ What is critical to understand, however, is not simply that a difference exists between physicians' understandings of resilience in themselves compared to in their colleagues, but rather to understand the content and potential impact of these differences.

Physicians' descriptions of their own resilience are in line with previous research, which suggests that physicians deny or avoid difficulties, opting instead to portray themselves as impervious by: 1) working through, ignoring, or denying stress; 2) concentrating on what they would do next; and 3) keeping stress to themselves. ${ }^{[41]}$ Medical training may exacerbate pre-existing characteristics such as altruism which may lead to physicians pushing themselves beyond normal human abilities, failure to recognize the unreasonable nature of the expectations they hold for themselves, or difficulty asking for help. ${ }^{[11]}$ Early in their careers, medical students' attitudes and behaviours may come to reflect the culture of medicine, which emphasizes the importance of prioritizing patients' needs ahead of one's own (even if the physician is sick), a sense of responsibility to one's colleagues, having selfless dedication to one's profession, remaining in control, having all the answers, and striving for perfection. ${ }^{[11,19,42-44]}$ In the highly competitive environment of medicine, physicians may develop attitudes centred around feeling immune to stress because of the emphasis on self-sufficiency, responsibility, and power ${ }^{[42]}$ Striving for perfection is in accordance with the idea of medicine being a calling or vocation and may be reinforced through society's expectations of physicians, further influencing physicians' own expectations. ${ }^{[20]}$ While it is important for physicians to be professional and strive for excellence in their work, the culture of medicine may, at times, foster unrealistic expectations and frame wellness as incongruent with professionalism. Holding high expecta- 
tions for oneself may be beneficial up to a certain point, after which these expectations may be overwhelming and lead to exhaustion or poor health.

As such, the way physicians described their own resilience may have consequences for their wellness. If physicians expect themselves to be immune to, push through, and rebound from stress, they may not develop effective coping strategies, ask for help, or accept help when it is offered. Instead, physicians' strategies may be unhealthy and potentially harmful. ${ }^{[45]}$ For example, keeping stress to oneself, concentrating on what to do next, and going on as if nothing happened are significantly related to emotional exhaustion in previous research. ${ }^{[41]}$ These strategies are similar to how participants described the feel of resilience, suggesting that physicians' views of their own resilience may be harmful. The way in which physicians described the look of resilience in their colleagues, however, presents a more realistic, achievable, and healthy approach. Raising awareness about more realistic expectations and self-appraisals regarding effective coping strategies, professionalism, and work performance may yield positive avenues for increasing physician resilience and wellness, as well as ensuring high quality care within our health systems.

This paper contributes to understandings of how physicians view resilience, highlighting important differences between physicians' perceptions of their own resilience and that of their colleagues. While previous research suggests that resilience may contribute to improved coping and wellness, this paper emphasizes that physicians may hold unrealistic and potentially unhealthy expectations for their own resilience.

These results should be interpreted in the context of the study design. There may be some self-selection bias where physicians who volunteered to participate in an interview hold different understandings or experiences of resilience or nonresilience than those who did not. While we are confident that the themes we describe here represent the perceptions of our participants, we are unable to conclude whether other physicians hold similar views. Despite this potential limi- tation, research has demonstrated that there are often few differences in the characteristics of responders compared to non-responders, suggesting that response or participation rates may not indicate biases. ${ }^{[46,47]}$ Future research with a larger, more representative sample would help to determine the extent to which physicians more broadly hold the views expressed by the participants in our study. Future research could also consider whether physicians who are perceived to be resilient actually feel resilient themselves. It is likely that some of the physicians described in this study as being resilient also felt resilient, but this was not within the scope of our project. We focused specifically on how our participants felt and how they viewed their colleagues, rather than how our participants were viewed by others. Furthermore, in response to additional research questions not presented here, our participants suggested that resilience is situational, contextual, and multidimensional rather than something that is fixed or static (results available from authors). Future studies should explore resilience not as an overarching absence or presence, but rather as a nuanced and dynamic experience of resilience or non-resilience. Finally, participants may have portrayed themselves as immune to stress in order to present a positive image that reflects the current culture of medicine. The results nevertheless illustrate the qualities physicians may perceive as desirable.

\section{Conclusion}

This study offers insight into how physicians understand and conceptualize resilience, in both themselves and their colleagues. While there were several similarities in how physicians described the look of resilience or non-resilience compared to the feel, physicians often held unrealistic expectations for themselves. Future research should examine how physicians' expectations regarding resilience are shaped and how they can become more realistic, as well as the implications these expectations may have. This knowledge can then be used in developing initiatives aimed at increasing physicians' awareness about more realistic expectations and self-appraisals and the risks of holding attitudes that emphasize being immune to stress.

\section{REFERENCES}

[1] Baird N, Fish JS, Dworkind M, et al. Physician, heal thyself. developing a hospital-based physician well-being committee. Can Fam Physician. 1995; 41: 259-63. PMid: 7734999.

[2] Cohen JS, Patten S. Well-being in residency training: a survey examining resident physician satisfaction both within and outside residency training and mental health in Alberta. BMC Med Educ. 2005; 5: 21-31. http://dx.doi .org/10.1186/1472-6920-5-21
[3] Linzer M, Gerrity M, Douglas JA, et al. Physician stress: results from the physician worklife study. Stress \& Health. 2002; 18(1): 37-42. http://dx.doi.org/10.1002/smi.917

[4] Dyrbye LN, West CP, Satele D, et al. Burnout among U.S. medical students, residents, and early career physicians relative to the general U.S. population. Acad Med. 2014; 89(3): 443-51. http: //dx.doi.org/10.1097/ACM.0000000000000134

[5] Shanafelt TD, Boone S, Tan L, et al. Burnout and satisfaction 
with work-life balance among US physicians relative to the general US population. Arch Intern Med. 2012; 172(18): 1377-85. http://dx.doi.org/10.1001/archinternmed.2012.3199

[6] Lin Y-W. The causes, consequences, and mediating effects of job burnout among hospital employees in Taiwan. J Hosp Adm. 2013; 2(1): $15-27$.

[7] Davis BH, Hill SEM, Fisher P, et al. Faculty burnout in a large department of pediatrics. J Hosp Adm. 2015; 4(2): 74-7. http: $/ / \mathrm{dx}$.doi.org/10.5430/jha.v4n2p74

[8] Thommasen HV, Lavanchy M, Connelly I, et al. Mental health, job satisfaction, and intention to relocate. opinions of physicians in rural British Columbia. Can Fam Physician. 2001; 47(4): 737-44.

[9] Hawton K, Clements A, Sakarovitch C, et al. Suicide in doctors: a study of risk according to gender, seniority and specialty in medical practitioners in England and Wales, 1979-1995. J Epidemiol Community Health. 2001; 55(5): 296-300. http://dx. doi .org/10.1136 /jech.55.5.296

[10] Hampton T. Experts address risk of physician suicide. JAMA. 2005; 294(10): 1189-91. http://dx.doi.org/10.1001/jama.294.10 .1189

[11] Privitera MR, Rosenstein AH, Plessow F, et al. Physician burnout and occupational stress: an inconvenient truth with unintended consequences. J Hosp Adm. 2015; 4(1): 27-35.

[12] Wallace JE, Lemaire JB, Ghali WA. Physician wellness: a missing quality indicator. Lancet. 2009; 374: 1714-21. http://dx.doi.o $\mathrm{rg} / 10.1016 / \mathrm{S} 0140-6736(09) 61424-0$

[13] Williams ES, Rondeau KV, Xiao Q, et al. Heavy physician workloads: impact on physician attitudes and outcomes. Health Serv Manage Res. 2007; 20(4): 261-9. http://dx.doi.org/10.1258/09514 8407782219067

[14] Arnetz BB. Psychosocial challenges facing physicians of today. Soc Sci Med. 2001; 52(2): 203-13. http://dx.doi.org/10.1016/S 0277-9536(00) 00220-3

[15] Haglund ME, aan het Rot M, Cooper NS, et al. Resilience in the third year of medical school: a prospective study of the associations between stressful events occurring during clinical rotations and student well-being. Acad Med. 2009; 84(2): 258-68. http://dx.doi.org/10.1097/ACM.0b013e31819381b1

[16] Zwack J, Schweitzer J. If every fifth physician is affected by burnout, what about the other four? resilience strategies of experienced physicians. Acad Med. 2013; 88(3): 382-9. http://dx.doi.org/10. 1097/ACM. 0b013e318281696b

[17] Chan AOM, Chan YH, Chuang KP, et al. Addressing physician quality of life: understanding the relationship between burnout, work engagement, compassion fatigue and satisfaction. J Hosp Adm. 2015; 4(6): 46-55. http://dx.doi.org/10.5430/jha.v4n6p46

[18] Dyrbye L, Shanafelt T. Nurturing resiliency in medical trainees. Med Educ. 2012; 46(4): 343. http://dx.doi.org/10.1111/j.136 5-2923.2011.04206.x

[19] Nedrow A, Steckler NA, Hardman J. Physician resilience and burnout: can you make the switch? FPM. 2013; 20(1): 25-30.

[20] Santomauro CM, Kalkman CJ, Dekker SWA. Second victims, organizational resilience and the role of hospital administration. J Hosp Adm. 2014; 3(5): 95-103. http://dx.doi.org/10.5430/jha.v 3n5p95

[21] Howe A, Smajdor A, Stöckl A. Towards an understanding of resilience and its relevance to medical training. Med Educ. 2012; 46: 349-56. http://dx.doi.org/10.1111/j.1365-2923. 2011 $.04188 . \mathrm{x}$

[22] Windle G. What is resilience? a review and concept analysis. Rev Clin Gerontol. 2011; 21: 152-69. http://dx.doi.org/10.1017 /S0959259810000420

Published by Sciedu Press
[23] Black C, Ford-Gilboe M. Adolescent mothers: resilience, family health work and health-promoting practices. J Advanced Nursing 2004; 48(4): 351-60. http://dx.doi.org/10.1111/j.1365-2 $648.2004 .03204 . x$

[24] Hunter AJ, Chandler GE. Adolescent resilience. Image: J Nurs Scholarship. 1999; 31(2): 243-47. http://dx.doi.org/10.1111/j.1 547-5069.1999.tb00488.x

[25] Werner EE. Resilient children. Young Child. 1984; 40: 68-72.

[26] Humphreys J. Research in sheltered battered women. Ment Health Nurs. 2003; 24(2): 137-52. http://dx.doi.org/10.1080/016 12840305293

[27] Christopher KA. Determinants of psychological well-being in Irish immigrants. Western J Nurs Res. 2000; 22(2): 123-42. http: //dx.doi.org/10.1177/019394590002200203

[28] Wagnild G. Resilience and successful aging: comparison among low and high income older adults. J Gerontol Nurs. 2003; 29(12): 42-9. http://dx.doi.org/10.3928/0098-9134-20031201-09

[29] Wenzell LB, Donnelly JP, Fowler JM, et al. Resilience, reflection, and residual stress in ovarian cancer survivorship: a gynecologic oncology group study. Psycho-Oncology. 2002; 11(2): 142-53 http://dx.doi.org/10.1002/pon.567

[30] Jensen PM, Trollope-Kumar K, Waters H, et al. Building physician resilience. Can Fam Physician. 2008; 54: 722-9. PMid: 18474706.

[31] Thomas SE, Haney MK, Pelic CM, et al. Developing a program to promote stress resilience and self-care in first-year medical students. Can Med Educ J. 2011; 2(1): e32-6. PMid: 21822458.

[32] Longenecker R, Zink T, Florence J. Teaching and learning resilience: building adaptive capacity for rural practice. a report and subsequent analysis of a workshop conducted at the rural medical educators conference, Savannah, Georgia, May 18, 2010. J Rural Health. 2012; 28: 122-7. http://dx.doi.org/10.1111/j.1748-0361.2011 $.00376 . \mathrm{x}$

[33] Epstein RM, Krasner MS. Physician resilience: what it means, why it matters, and how to promote it. Acad Med. 2013; 88: 382-9. http://dx.doi.org/10.1097/ACM. Ob013e318280cff0

[34] Sood A, Prasad K, Schroeder D, et al. Stress management and resilience training among department of medicine faculty: a pilot randomized clinical trial. J Gen Intern Med. 2011; 26(8): 858-61. http://dx.doi.org/10.1007/s11606-011-1640-x

[35] Sandelowski F. Sample size in qualitative research. Res Nurs Health 1995; 18: 179-183. http://dx.doi.org/10.1002/nur.47701 80211

[36] Guest G, Bunce A, Johnson L. How many interviews are enough? an experiment with data saturation and variability. Field Method. 2006; 18(1): 59-82. http://dx.doi.org/10.1177/1525822X0 5279903

[37] Berg BL, Lune H. Qualitative research methods for the social sciences. $8^{\text {th }}$ ed. Boston, MA: Pearson; 2012.

[38] Malle BF. The actor-observer asymmetry in attribution: a (surprising) meta-analysis. Psychol Bull. 2006; 132(6): 895-919. http: //dx.doi.org/10.1037/0033-2909.132.6.895

[39] Sande GN, Goethals GR, Radloff CE. Perceiving one's own traits and others': the multifaceted self. J Pers Soc Psychol. 1988; 54(1): 13-20. http://dx.doi.org/10.1037/0022-3514.54.1.13

[40] Brown JD. Evaluations of self and others: self-enhancement biases in social judgments. Soc Cognition. 1986; 4(4): 353-76. http: //dx.doi.org/10.1521/soco.1986.4.4.353

[41] Lemaire JB, Wallace JE. Not all coping strategies are created equal: a mixed methods study exploring physicians' self reported coping strategies. BMC Health Serv Res. 2010; 10: 208-17. http: //dx.doi.org/10.1186/1472-6963-10-208 
[42] Paolini H. Inside the mind of a physician: illuminating the mystery of how doctors think, what they feel, and why they do the things they do. Orlando, FL: Florida Hospital; 2009.

[43] Silbaugh B, Winchester-Silbaugh J. Physician resiliency? Physician Exec. 1998; 24(4): 30-2. PMid: 10186381.

[44] Thompson WT, Cupples ME, Sibbett CH, et al. Challenge of culture, conscience, and contract to general practitioners' care of their own health: qualitative study. BMJ. 2001; 323: 728-31. http: //dx.doi.org/10.1136/bmj.323.7315.728
[45] Wallace JE, Lemaire JB. Physician coping styles and emotional exhaustion. Ind Relat. 2013; 68: 187-209. http://dx.doi.org/10. 7202/1016316ar

[46] Cummings SM, Savitz LA, Konrad TR. Reported response rates to mailed physician questionnaires. Health Serv Res. 2001; 35(6): 1347-55. PMid: 11221823.

[47] James KM, Ziegenfuss JY, Tilburt JC, et al. Getting physicians to respond: the impact of incentive type and timing of physician survey response rates. Health Serv Res. 2011; 46(1 Pt 1): 232-42. http://dx.doi.org/10.1111/j.1475-6773.2010.01181.x 